\title{
ORT_20 - Epitope signatures in COVID-19 patients
}

Tatjana Schwarz ${ }^{1}$; Kirsten Heiss ${ }^{2 *}$; Florian Kurth ${ }^{3}$; Leif Sander ${ }^{3}$; Clemens-Martin Wendtner; Manuela A. Hoechstetter ${ }^{4}$; Marcel A. Müller ${ }^{1}$; Christian Drosten ${ }^{1}$; Volker Stadler²; Victor M. Corman ${ }^{1}$.

${ }^{1}$ Institute of Virology, Charité-Universitätsmedizin Berlin, corporate member of Freie Universität Berlin, Humboldt-Universität zu Berlin, and Berlin Institute of Health, Berlin, Germany;

${ }^{2}$ PEPperPRINT GmbH, Heidelberg, Germany;

${ }^{3}$ Department of Infectious Diseases and Respiratory Medicine, Charité - Universitätsmedizin Berlin, Corporate Member of Freie Universität Berlin, Humboldt-Universität Zu Berlin, and Berlin Institute of Health, Berlin, Germany;

${ }^{4}$ Munich Clinic Schwabing, Academic Teaching Hospital, Ludwig-Maximilians University (LMU), Munich, Germany.

Introduction: The worldwide ongoing transmission of COVID-19 is a major global health concern. The causative agent of this acute respiratory disease is a newly emerged coronavirus named SARS-CoV-2. The virus originated from China in late 2019 and rapidly spread across the globe. The course of the disease ranges from non-symptomatic to mild symptoms such as fever and cough to severe cases with pneumonia, acute respiratory distress and potentially death. Humoral responses are an important defense mechanism in viral infections. The investigation of antigens and/or epitopes recognized by SARS-CoV-2-specific antibodies is not only crucial for the development of intervention strategies, but also for epidemiological studies, disease prognosis and the identification of novel diagnostic markers.

Objective: The goal of this study was a comprehensive analysis of the epitope-specific antibody responses across the entire proteome of SARS-CoV-2 in order to identify potential discriminating serological markers for different COVID-19 disease phases (acute and convalescent phase) and outcomes (mild and severe), respectively.

Methodology: High-density peptide microarrays are a great tool to screen large libraries of peptides against serum antibodies. We screened sera from COVID-19 patients in conjunction with high-density peptide microarrays covering the entire proteome of SARS-CoV-2 as 15 amino acid peptides with an overlap of 13 amino acids. The high peptide-to-peptide overlap of our SARS-CoV-2 proteome array allowed a high-resolution epitope analysis giving a detailed picture of antibody binding patterns. We investigated antibody profiles (i) longitudinally in COVID-19 with mild disease symptoms and (ii) in patients with a mild versus severe COVID-19 disease progression. We applied a dual isotype read-out, analysing IgG and IgA specific antibody responses against SARS-CoV-2.

Results: Monitoring the longevity of epitope-specific antibody responses in COVID-19 patients demonstrated increasing IgG antibody reactivities over time while IgA-specific epitope responses peaked in the early convalescent phase before declining until around week 10 post symptom onset for most of the epitopes. Our study identified a NSP15-derived peptide as potential marker of acute, early and late convalescent mild COVID-19 disease. Moreover, we discovered several IgG and/or IgA-specific epitopes, which were significantly associated with severe COVID-19 disease.

Conclusion: The herein identified epitopes may serve as biomarkers for early and/or late COVID-19 disease detection and as serological markers able to discriminate severe from mild disease courses. Due to the power of peptide microarrays to map antibody interactions at amino acid level, the technology may ideally suit to investigate antibody responses against newly identified mutations of SARS-CoV-2.

Keywords: SARS-CoV-2 Corona Virus; Peptide Microarrays; Biomarker Discovery 